\title{
Transcutaneous oxygen saturation in sleeping infants: prone and supine
}

\author{
S Levene, S A McKenzie
}

\begin{abstract}
Pulse oximetry was used to measure transcutaneous arterial oxygen saturation in infants aged 2 to 11 months prone and supine in quiet sleep. Groups of healthy infants $(n=34)$, infants with upper respiratory tract infections $(n=13)$, and infants with generalised moderately severe lower respiratory tract infections $(n=17)$ were studied. No clinically important differences were demonstrated in any of these groups, although there was a small advantage in the prone position in the group with lower respiratory tract infection. The effect of posture on infants with more severe lower respiratory tract infection and during active sleep has yet to be determined.
\end{abstract}

The incidence of the sudden infant death syndrome (SIDS) has been linked to sleeping posture. ${ }^{1-5}$ Studies of arterial oxygen tension in sick adults and neonates have shown that it may be altered with change in posture. ${ }^{6-8}$ If arterial oxygen tension in infants is improved by adopting either the prone or the supine position, there may be implications for the prevention of SIDS and the care of infants with and without upper and lower respiratory tract infections. It is difficult to assess arterial oxygen tension noninvasively in infants. Transcutaneous oxygen tension reflects this but requires careful calibration, a heated probe (which may burn the skin), ${ }^{9}$ and is considered by some to be less reliable beyond the neonatal period. ${ }^{10}$ The measurement of arteral oxygen saturation by pulse oximetry requires no calibration, is completely non-invasive, and has been shown to agree within acceptable limits with arterial oxygen saturation measured by co-oximetry over its normal range for infants. ${ }^{11-13}$ This study used this convenient method. Its purpose was to determine whether there was any clinically relevant difference in arterial oxygen saturation in sleeping infants between the prone and supine position.

\section{Subjects and methods}

Parents of all infants entered gave informed consent. The study received ethical committee approval.

The healthy infants studied were well and aged 2 to 11 months. They were recruited through the lying in wards and well baby clinics and did not have cardiorespiratory disorders or respiratory tract infections. Measurements were carried out by day in a specially reserved room in the neonatal unit or in the infants' own homes. Infants were soothed to sleep in their normal sleep position. The position for the study was randomised before knowledge of the infant's normal sleep position. The infants were turned when asleep if necessary. After a minimum of 10 minutes stabilisation arterial oxygen saturation was measured for a continuous period of 20 minutes. All patients were studied during quiet sleep. ${ }^{14}$ This both ensured that no corrections were required for sleep state ${ }^{15}$ and minimised the number of recorded artefacts in the arterial oxygen saturation due to movement. All measurements were taken on a Biox 3700 (software revision $M$ ) pulse oximeter with a neonatal flexprobe on the infant's foot, toe, or hand (Ohmeda). This has an accuracy of $1.5 \%$ (1 SD) in the range studied..$^{13}$ During recording the plethysmographic waveform displayed was continuously observed for movement artefact, as demonstrated by irregularity of the normal pulse waveform. Any artefactual data points were eliminated from the subsequent analysis. Data were stored in the oximeter memory and subsequently off loaded by computer; the sampling interval for data points was 20 seconds. The age of the subjects was such that no correction for fetal haemoglobin was required.

It was calculated in advance that 24 infants were required in each limb to detect a difference of $3 \%$ in arterial oxygen saturation with a 0.01 risk of a false positive result (type I error) and a 0.2 risk of a false negative result (type II error). A value for the SD of the expected arterial oxygen saturation (3\%) was obtained from Mok and colleagues. ${ }^{15}$ Interim analysis was carried out when $70 \%$ of subjects were enrolled.

The study of infants with upper respiratory tract infection closely resembled the study of healthy infants but the subjects were previously well infants with upper respiratory tract infection and nasal discharge. In this part of the study, each infant was studied both prone and supine, acting as its own control. Numbers required would thus be fewer than the first study. It was elected to study about 12 babies in the first instance.

The third group comprised previously well infants admitted for inpatient treatment to a children's ward with the generalised lower respiratory tract infection of bronchiolitis as determined clinically by tachypnoea, fever, wheezes, and crackles on auscultation, with no focal signs either on clinical examination or on chest radiography. Each infant acted as its own control and was studied breathing air. It was decided not to study any baby with an arterial 


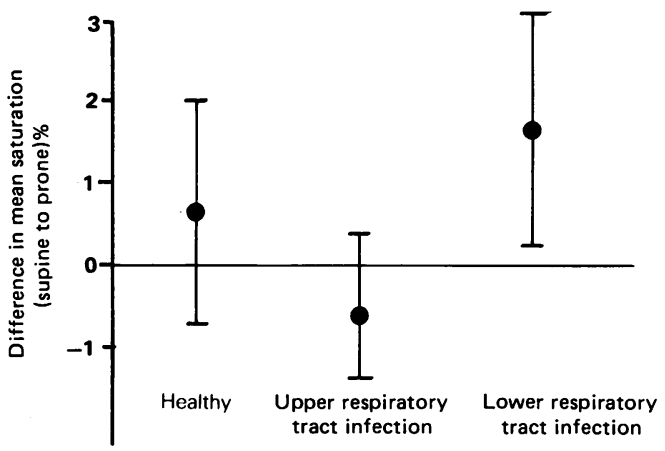

Difference between sample mean arterial oxygen saturation (\%) supine and prone for the three groups of infants (mean, $95 \%$ confidence intervals).

oxygen saturation of less than $85 \%$ in air. A pilot study suggested there would be a bigger variability in the difference in arterial oxygen saturation between the two positions in this group and so it was decided to study about 20 patients in the first instance.

\section{Results}

Forty two healthy infants were entered into the study: eight infants could not be studied as they did not go to sleep or woke when turned to the predetermined sleep position. Of those studied 17 infants were studied sleeping prone and 17 supine; there were 17 boys and 17 girls.

Values for arterial oxygen saturation observed were: prone infants mean (SD) $94 \cdot 8 \%(2 \cdot 0)$ and supine infants $94 \cdot 1 \%(1 \cdot 8)$. The values were normally distributed by the Shapiro Francia W' test $\left(W^{\prime}=0 \cdot 99\right)$. The difference between the sample mean arterial oxygen saturation in prone and supine infants was $0.7 \%$ in favour of the prone position with a $95 \%$ confidence interval from -0.7 to $2.0 \%$ (figure); the $t$ test statistic was 1.03 , with 32 degrees of freedom $(p=0.3)$.

Seventeen infants with upper respiratory tract infection were recruited and 13 were successfully studied; there were seven boys and six girls. Values for arterial oxygen saturation observed were prone infants mean (SD) $93 \cdot 1 \%$ $(2 \cdot 7)$ and supine infants $93.6 \%(2 \cdot 7)$. These values were normally distributed $\left(W^{\prime}=0.98\right)$. The mean difference between the prone and supine positions was $-0.6 \%$ with $95 \%$ confidence interval from -1.4 to $0.4 \%$ (figure); the $t$ test statistic was $-1 \cdot 28$ with 12 degrees of freedom $(p=0 \cdot 23)$.

Twenty two infants with lower respiratory tract infection were recruited and 17 were successfully studied; there were 12 boys and five girls. Values for arterial oxygen saturation observed were prone infants mean (SD) $90.5 \%$ $(2 \cdot 9)$ and supine infants $88.7 \%(4 \cdot 6)$. These values were normally distributed $\left(W^{\prime}=0.98\right)$. The mean difference between the prone and supine positions was $+1.7 \%$ with $95 \%$ confidence interval from $0.3 \%$ to $3.2 \%$ (figure); the $t$ test statistic was 2.54 with 16 degrees of free$\operatorname{dom}(p=0.02)$.

For all three studies the SD of the mean arterial oxygen saturation within each study in each position was calculated. The values were within a range of $0.4-1 \cdot 5 \%$ and there was no difference between studies or positions.

\section{Discussion}

This study confirms previous work on normal values for arterial oxygen saturation including the normal range. ${ }^{15}$ It failed to detect a difference in arterial oxygen saturation with position in normal sleeping infants or those with an upper respiratory tract infection; in those with a lower respiratory tract infection a difference of $1 \cdot 7 \%$ (confidence interval $0 \cdot 3 \%$ to $3 \cdot 2 \%$ ) was detected. This represents a difference of $1 \mathrm{kPa}$ at the most in arterial oxygen tension, or $7 \%$ in oxygen content, and even less if the initial arterial oxygen saturation lay on the steep part of the oxygen dissociation curve. ${ }^{16}$

The healthy infants formed a group with low variability in sleeping saturation. Recruitment of large numbers was easy, and so a parallel study, less tedious to patients and their parents, could be undertaken. The results indicate that a clinically important difference would not have been detected even if each patient had been used as its own control. Saturations of the infants with upper and lower respiratory tract infections were far more variable and numbers available for recruitment were smaller; thus it was appropriate to study each infant both prone and supine on a single day.

For healthy infants and infants with an upper respiratory tract infection, the study indicates that sleeping posture in quiet sleep is not relevant to arterial oxygen saturation. For infants with moderately severe lower respiratory tract infection this study indicates that although arterial oxygen saturation is greater in the prone position, possibly because of better ventilation/ perfusion matching, the difference is not of a magnitude likely to be of clinical importance. Neonates and adults, however, with more severe respiratory disease, ${ }^{6-8}$ have shown more noticeable improvement in the prone position and it may be that this could also be true for infants with more severe respiratory disease. The study was limited to quiet sleep. Beyond the age of 1 month it has been shown in babies in the supine position that arterial oxygen saturation is lower in active sleep but by less than $1 \% .{ }^{15}$ Whether the prone position confers similar or greater change in arterial oxygen saturation during active sleep has yet to be determined.

This work was funded by a grant from the North East Thames Regional Health Authority.

1 Lee NYA, Chan YF, Davies DP, Lau E, Yip DCP. Sudden infant death syndrome in Hong Kong: confirmation of low incidence. $\mathrm{Br}$ Med $\mathcal{F}$ 1989;298:721.

2 de Jonge GA, Engleberts AC, Koomen-Liefting AJM, Kostense PJ. Cot death and prone sleeping position in the Netherlands. Br Med $\mathcal{f}$ 1989;298:722.

3 Emery JL. Epidemiology of 'sudden, unexplained or rapid' deaths in children. $\mathrm{Br} M e d \mathcal{F} 1959$;ii:925-8.

4 Kahn A, Blum D, Hennart P, et al. A critical comparison of the history of sudden-death infants and infants hospitalised for near-miss for SIDS. Eur $\mathcal{f}$ Pediatr 1984;143:103-7.

5 Beal S. Sleeping position and SIDS. Lancet 1988;ii:512.

6 Douglas WW, Rehder K, Beynen FM, et al. Improved oxygenation in patients with acute respiratory failure: the prone position. Am Rev Resp Dis 1977;115:559-66.

7 Martin RJ, Herrell N, Rubin D, et al. Effect of supine and 
prone positions on arterial oxygen tension in the preterm infant. Pediatrics 1979;63:528-31.

8 Wagaman MJ, Shutack JG, Moomjian AS. Improved oxygenation and lung compliance with prone positioning of neonates. $\mathcal{F}$ Pediatr 1979;94:787-91.

9 Jennis MS, Peabody JL. No burns, no gradient: pulse oximetry, an alternative to transcutaneous $\mathrm{PO}_{2}$. Pediatr Res 1983;17:374A.

10 Solimano AJ, Smyth JA, Mann TJ, Albersheim SG, Lockitch G. Pulse oximetry, advantages in infants with bronchopulmonary dysplasia. Pediatrics 1986;78:844-9.

11 Durand M, Ramanathan R. Pulse oximetry for continuous oxygen monitoring in sick newborn infants. F Pediatr 1986; 109:1052-6.

12 Fanconi S, Doherty P, Edmonds JF, Barker GA, Bohn DJ.
Pulse oximetry in pediatric intensive care: comparison with measured saturations and transcutaneous oxygen tension. 7 Pediatr 1985;107:362-6.

13 Shippy MB, Peterson MT, Whitman RA, Shivers CR. A clinical evaluation of the BTI Biox ear oximetry. Respiratory Care 1984;29:730-5.

14 Prechtl HFR. The behaviour states of the newborn infant (a review). Brain Res 1974;76:185-212.

15 Mok JYQ, McLaughlin FJ, Pintar M, et al. Transcutaneous monitoring of oxygenation: what is normal? $\mathcal{f}$ Pediatr 1986;108:365-71.

16 Delivera-Papadopoulos M, Roncevic NP, Oski F. Postnatal changes in oxygen transport of term, premature and sick changes in oxygen transport of term, premature and sick adult hemoglobin. Pediatr Res 1971;5:235-45.

\section{Forty years ago}

\section{Pulmonary collapse in pertussis}

In the five year period 1943-7 the average annual notifications of pertussis in England and Wales were 87600 with an average of 890 deaths.

In order to follow the course of respiratory complications in pertussis a prospective study was undertaken of 44 children aged from 6 months to 8 years whose attack of whooping cough was serious enough for them to be admitted to the Kent County Hospital. ${ }^{1}$ All but four of the children were considered to have moderate or severe disease and four died. Clinical and radiographic examinations were carried out at frequent intervals and the patients were followed up either until the lungs were clear or for a minimum of one year.

Lobar or segmental collapse was seen in 39 cases $(89 \%)$, including three of the four mild cases. The right middle and lower lobes were more commonly affected than the lingula and left lower lobe. Over $70 \%$ of collapsed lobes had re-expanded within nine months but there was no further expansion after this time. Seven of the 33 children followed up for more than one year $(17 \cdot 5 \%$ of the 40 survivors) showed evidence of persistent collapse and were therefore likely to develop bronchiectasis. The author suggests that many cases of prolonged collapse after an attack of pertussis were being missed and that the incidence of bronchiectasis as a complication of pertussis was probably even greater than $17 \cdot 5 \%$.

\section{Gastroenteritis}

In the City of Aberdeen in 1946 the infant mortality rate was 50 (/1000 live births). In 1947 this figure rose to 64 due almost entirely to an epidemic of gastroenteritis in which 105 of 207 cases died.

The report of the epidemic showed the highest incidence to be in babies aged 2 months and some $90 \%$ of the cases were under the age of 7 months. ${ }^{2}$ However, mortality rates were similar in each monthly age group in the first year of life. There were two peaks of incidence during the year, in April and July. Only $2 \%$ of affected babies were fully breast fed. Almost $60 \%$ of cases acquired the disease in hospital or some other institution and there were six outbreaks in children's wards.

There was no evidence that a virus was responsible for the epidemic but stool cultures from $95 \%$ of affected babies grew Bacterium coli (sic) neopolitanum. This organism was found in $3.7 \%$ of children with diarrhoea from other causes and in $1.8 \%$ of controls without diarrhoea. $B$ coli beta was grown in a number of cases towards the end of the epidemic. However, the authors were not prepared to draw any definite conclusions about the causative agent of the epidemic.

'Arobon', a powder prepared from the bean of the carob tree (Ceratonia siliqua) and mixed with starch and cocoa was first used to treat infantile gastroenteritis during the Spanish civil war in the late 1930s. A similar preparation based on the carob bean is 'Nestargel', still sometimes used to thicken infant feeds. The action of Arobon in gastroenteritis is said to be due to its high power of absorption of fluid and toxic substances.

Several uncontrolled trials of this preparation in cases of infantile gastroenteritis were reported in the late 1940s and a controlled trial was carried out at the Hospital for Sick Children, Great Ormond Street. ${ }^{3}$ Alternate cases admitted to the gastroenteritis unit were given either standard treatment (nil by mouth for 24 hours, half strength Hartmann's solution by mouth or intravenously depending on severity, followed by gradual introduction of milk feeds) or standard treatment with Arobon in water in place of the oral Harmann's solution and added to the milk feeds. Over a period of six months there were 25 cases in the control and treated groups which were comparable in age, severity, and incidence of parenteral infection.

There was no apparent difference between the two groups (no statistical analysis was made) in average duration of stay or average time for the stools to reduce to four/day, although Arobon did seem to control quickly the number of stools in four mild cases not associated with parenteral infection. Older infants often disliked the taste of Arobon and in some babies it aggravated the tendancy to vomit.

1 Nicholson DP. Pulmonary collapse in pertussis. Arch Dis Child 1949;24:29-40.

2 Giles C, Sangster G, Smith J. Epidemic gastro-enteritis of infants in Aberdeen during 1947. Arch Dis Child 1949;24: $45-53$.

3 Beynon D. Arobon in the treatment of infantile gastroenteritis: a clinical trial. Arch Dis Child 1949;24:41-6.

A D M JACKSON 